\title{
Research Priorities for Preventing Unsafe Abortions in the WHO Africa Region
}

\author{
Leopold Ouedraogo ${ }^{*}$, Triphonie Nkurunziza ${ }^{1}$, Assumpta Muriithi ${ }^{1}$, Chilanga Asmani ${ }^{1}$, \\ Hayfa Elamin', Souleymane Zan1, Mihretu Belete1, Gbenou Dina1, Theopista Kabuteni John', \\ Bigirimana Françoise1, Dadji Kwami', Kim Caron Rahn², Ali Moazzam², Tolu Lemi², Blami Dao3, \\ Issiaka Sombie ${ }^{4}$, Okech Mollent ${ }^{5}$
}

\author{
${ }^{1}$ Reproductive, Maternal Health and Ageing, WHO Regional Office for Africa, Brazzaville, Congo \\ ${ }^{2}$ Department of Reproductive Health and Research, World Health Organization, Geneva, Switzerland \\ ${ }^{3}$ JHPIEGO, Ouagadougou, Burkina Faso \\ ${ }^{4}$ West Africa Health Organization, Ouagadougou, Burkina Faso \\ ${ }^{5}$ Peak Moments Global HR Solutions, Nairobi, Kenya \\ Email: `ouedraogol@who.int, nkurunzizat@who.int, kimca@who.int, muriithia@who.int, asmanic@who.int, elaminha@who.int, \\ alimoa@who.int, tolul@who.int, blami.dao@jhpiego.org, zans@who.int, tafesseb@who.int, Kabutenit@who.int, \\ bigirimanaf@who.int, dadjik@who.int, isombie@wahooas.org, mollentakinyi@gmail.com
}

How to cite this paper: Ouedraogo, L., Nkurunziza, T., Muriithi, A., Asmani, C., Elamin, H., Zan, S., Belete, M., Dina, G., John, T.K., Françoise, B., Kwami, D., Rahn, K.C., Moazzam, A., Lemi, T., Dao, B., Sombie, I. and Mollent, O. (2021) Research Priorities for Preventing Unsafe Abortions in the WHO Africa Region. Advances in Reproductive Sciences, 9, 24-32. https://doi.org/10.4236/arsci.2021.91003

Received: October 24, 2020

Accepted: December 27, 2020

Published: December 30, 2020

Copyright $\odot 2021$ by author(s) and Scientific Research Publishing Inc. This work is licensed under the Creative Commons Attribution International License (CC BY 4.0).

http://creativecommons.org/licenses/by/4.0/

(c) (i) Open Access

\begin{abstract}
Background: Unsafe abortion is a commonly neglected sexual and reproductive health and rights issue despite the serious health problems it causes to women and girls in their reproductive ages. It is classified as a main cause of maternal mortality and morbidity. This paper has considered questions that have the greatest potential to successfully reduce unsafe abortions in the resource poor settings. Methods: We adapted the Child Health and Nutrition Research Initiative (CHNRI) to identify and prioritize many competing sexual and reproductive health and rights research ideas that impact the health of the populations. The implementation was done in three phases which included generation and collection of research ideas from various experts virtually in August 2019 consolidation of the potential questions through thematic analysis conducted in September 2019. Finally, scoring and ranking of the research questions was done in a workshop of experts. Results: Out of a list of 45 priority research questions, two questions were ranked the highest scoring 28 out of the possible 30 . The research priorities include: "The effectiveness of interventions (e.g. counseling or incentives or home visits) to increase post abortion uptake and continuance", "Reducing repeat abortion on improving maternal health outcomes" and "Evaluation of community-based awareness programs to reduce unwanted pregnancies and encourage women to seek help early". Conclusions: Ten key research priorities in preventing unsafe abortion were identified. The priority list covers areas of focus that
\end{abstract}


could effectively impact preventing unsafe abortions while also acting as a knowledge base for researchers, policy makers and other interested stakeholders who would want to invest in this area.

\section{Keywords}

Unsafe Abortion, Post-Abortion Contraception, Sexual and Reproductive Health, Unplanned, Abortion

\section{Introduction}

It is estimated that an average of 73.3 million women and girls induced safe and unsafe abortions between 2015 and 2019 globally. According WHO reports, there were about 39 abortions conducted out of every 1000 women whose ages range between 15 and 45 years. Out of these, one out of every three abortions are carried out in unsafe environments [1]. Abortion is defined by the World Health Organization (WHO) as the "termination of an unintended pregnancy either by persons lacking the required skills or in an environment lacking the minimal medical standards, or both" [2]. Abortion is one of the leading causes of maternal death in low- and middle-income countries [3]. Unsafe abortion is a commonly neglected reproductive health care problem in developing countries, yet it poses a serious threat to the health of millions of women during their reproductive lives [4]. Until unsafe abortion and its consequences are eliminated, complications from unsafe abortion will remain a major cause of maternal mortality and morbidity.

Unsafe abortion is one of the mostly neglected reproductive health care problems especially in the developing countries, thereby increasing the impact of maternal mortality and morbidity for millions of women and girls in their reproductive lives. Of these unsafe abortions between $4.7 \%$ and $13.2 \%$ lead to maternal deaths [3] [5]. Sadly, the women in Africa are at a higher risk of dying from both safe and unsafe abortions [6]. Where legal services are readily accessible and available, abortions are generally safe compared to where access and availability of legal services are highly restricted [7] [8]. Abortion laws and services are needed to protect the health and human rights of all women, including adolescents to avoid situations that lead women and adolescents to seek unsafe abortion [9].

The 2019 International Conference on Population and Development (ICPD) outlined the issues and challenges for work in the area of unsafe abortion and urged governments, health providers and civil society to take major steps to advance women's and girls' sexual and reproductive rights, in line with international human rights standards and public health guidance, including liberalizing abortion laws in countries that can revolutionize access and improved safety of abortion around the world [10] [11] [12]. Despite global health commitments, such as the Sustainable Development Goals (SDGs) and the Global Strategy for Women's, Children's and Adolescent's Health (2016-30), the Guttmach- 
er-Lancet Commission has documented continued gaps in sexual and reproductive health and rights. In this context, timely evidence for the incidence of unintended pregnancy and abortion can motivate investment and greater commitment to increase access to services and inform policies and programmes [13].

Sexual reproductive and health rights stakeholders are confronted with new ethical challenges along with global development and population demands which require more investigations. Scarcity and unreliability of data to programmatically respond is also an issue. Moreover, the limited financial and human resources are more pressing in developing countries and have major influence planning and implementing research [14]. Due to this, the WHO African Region, sought to conduct a research prioritization exercise to identify key areas of focus in relation to unsafe abortion. Until interventions arise from such valid prioritization of unsafe abortion that informs policy and direction to countries, complications from unsafe abortion will remain a major cause of maternal mortality and morbidity.

\section{Methods}

This paper reports part of a larger research prioritization exercise conducted for the WHO African region. The paper focuses on the thematic area of SRHR on preventing unsafe abortion. The research prioritization adopted Child Health and Nutrition Research Initiative (CHNRI approach [15]. Questions identified were based on their answerability (can the questions be ethically answered), effectiveness (can it result to effective intervention), deliverability (is it deliverable, acceptable and affordable), potential impact (address the challenges of unsafe abortion) and equity (can it reach the most vulnerable). The prioritization exercise was implemented in three phases: 1) the generation and collection of research questions, 2) consolidation of research questions and thematic analysis, and 3) the prioritization exercise of the research questions using pre-defined scoring criteria. In the first phase an online survey to stakeholders with diverse experiences identified potential research questions on SRHR. The questions from the online survey were received for technical analysis by a team at WHO HQ. The online survey built on the results from a prior research prioritization exercise conducted by EMRO and AFRO in 2016 [16]. The priority questions consolidated into 12 themes areas for the next step of the process.

Phase 2 and 3 of the research prioritization process were conducted in a meeting held in Cape Town, South Africa from 29th October to 1st November, 2019. A total of 67 SRHR experts responsible for SRHR programmes from Ministries of health, partners supporting SRHR in the African region and WHO technical officers all from SRHR priority countries. A list of 45 research questions were reviewed with an aim of identifying duplications for merging, out of scope questions and those that had inconsistences in wordings for review. The process generated a list of 23 priority areas. In phase 3, a team of six experts using a modified ranking criteria, scored the final list of research questions against 
six distinct areas namely magnitude, severity, effectiveness, feasibility, burden and equity. The least score in the scoring criteria was 1 while the highest score was 5 . Each question could therefore attain a lowest score of 6 or highest score of 30. Table 1 presents the modified scoring criteria used. No special weighting was applied to the scoring criteria.

\section{Results}

In this paper, we report the results of research prioritization exercise on sexual and reproductive health and rights with a focus on preventing unsafe abortion. A total of 67 experts out of the 80 invited participated in the research prioritization process thus a response rate of $83.7 \%$. The aim of this exercise was to identify key research questions on preventing unsafe abortion that would guide the WHO Africa Region research agenda.

The thematic area on preventing unsafe abortion generated a total of 45 research questions from the online survey. The questions were systematically reviewed by a team of experts who on consensus agreed on 23 key questions to be subjected to scoring and ranking. Table 2 presents the scoring and distribution of the questions on preventing unsafe abortion. Two questions were ranked highest and scored 28 out of the possible 30 points. They are "evaluate the effectiveness of interventions (e.g. counselling or incentives, or home visits) to increase post-abortion contraception uptake and continuance, and reduce repeat abortion, on improving maternal health outcomes" and "evaluation of community-based awareness programs to reduce unwanted pregnancies and encourage women to seek help early". Four questions scored 27 points. These included "community and client's perception towards accessible safe and post abortion services"; "evaluation of the quality of safe abortion and post abortion care services"; "evaluation of the effectiveness and feasibility of implementing current

Table 1. Modified scoring criteria.

\begin{tabular}{|c|c|c|}
\hline Criteria & Definition & Scoring \\
\hline 1) Magnitude & $\begin{array}{l}\text { Magnitude of the problem; in terms of the proportion of the population, such as women, under } 5 \text { children, } \\
\text { elderly, are affected. }\end{array}$ & $1-2-3-4-5$ \\
\hline 2) Severity & $\begin{array}{l}\text { Of the condition; i.e. danger to the individual and the community. How serious is the condition. Does it } \\
\text { threaten life, cause major suffering, and decrease the ability to lead a normal life. }\end{array}$ & $1-2-3-4-5$ \\
\hline 3) Effectiveness & $\begin{array}{l}\text { Based on the best existing evidence and knowledge, would intervention be efficacious in reducing disease } \\
\text { burden? It is likely to be effective under programme conditions. }\end{array}$ & $1-2-3-4-5$ \\
\hline 4) Feasibility & $\begin{array}{l}\text { Taking into account a) the infrastructure and resources required to deliver effective interventions (e.g. } \\
\text { human resources, health facilities, communication and transport infrastructure), and b) the need for } \\
\text { change in demand, beliefs and attitudes of users, would you say that the endpoints of the research would } \\
\text { be deliverable? affordability and sustainability. }\end{array}$ & $1-2-3-4-5$ \\
\hline 5) Burden & $\begin{array}{l}\text { Diseases burden reduction; taking into account the best available information, would you say that reaching } \\
\text { of research endpoints would eventually, have a "capacity" to impact directly and indirectly disease burden. } \\
\text { E.g. up to } 5 \% \text { to } 10 \% \text { reduction in long run. }\end{array}$ & $1-2-3-4-5$ \\
\hline 6) Equity & $\begin{array}{l}\text { Equity enhancing; does the intervention affect mainly the underprivileged in the population? Intervention } \\
\text { has potential to improve equity in disease burden distribution in the longer term? }\end{array}$ & $1-2-3-4-5$ \\
\hline
\end{tabular}

1 is the lowest score and 5 the highest score. Each question could therefore attain a lowest score of 6 or highest score of 30 . 
Table 2. Research priorities and their scores.

1) Evaluate the effectiveness of interventions (e.g. counselling or incentives, or home visits) to increase post-abortion contraception uptake and continuance, and reduce repeat abortion, on improving maternal health outcomes.

2) Evaluation of community-based awareness programs to reduce unwanted pregnancies and encourage women to seek help early.

3) Community and client's perception towards accessible safe and post abortion services.

4) Evaluation of the quality of Safe Abortion and post abortion care services.

5) Evaluation of the effectiveness and feasibility of implementing current guidelines on safe abortion services.

6) Evaluation of the impact of integrating safe and post abortion services in to routine reproductive health care services.

5

$$
4
$$

5

7) Perspectives of providers and clients on the law governing provision of abortion services.

\begin{tabular}{|c|c|c|c|c|c|c|}
\hline 4 & 5 & 4 & 5 & 5 & 5 & 28 \\
\hline 4 & 5 & 4 & 5 & 4 & 5 & 27 \\
\hline 4 & 5 & 4 & 5 & 5 & 4 & 27 \\
\hline 4 & 4 & 5 & 5 & 5 & 4 & 27 \\
\hline 5 & 4 & 5 & 4 & 4 & 5 & 27 \\
\hline 5 & 4 & 4 & 5 & 3 & 4 & 25 \\
\hline 5 & 4 & 4 & 3 & 4 & 4 & 24 \\
\hline 4 & 4 & 4 & 2 & 4 & 4 & 22 \\
\hline 4 & 4 & 3 & 3 & 4 & 3 & 21 \\
\hline
\end{tabular}

8) Effectiveness, safety and cost of working with other sociocultural organizations in safe abortion services.

9) Evaluating approaches that work to reduce unsafe abortion among adolescent girls and young women.

10) Impact of mHealth - use of digital technology to increase knowledge and access to safe abortion services.

guidelines on safe abortion services"; and, "evaluation of the impact of integrating safe and post abortion services in to routine reproductive health care services". The least score question with 21 points was on the "impact of mHealth - use of digital technology to increase knowledge and access to safe abortion services".

\section{Discussion}

Unsafe abortion is among the causes of maternal mortality and morbidity in countries with restrictive abortion laws [17] [18]. The research topics identified will guide in what needs to be done to reduce unsafe abortion and its consequences. The unmet need for contraception remains high in many low-resource countries [19]. This priority setting exercise identified top priority research questions which will go a long way in preventing unsafe abortions. The highly ranked priority questions include interventions which advocate for increased uptake of post-abortion contraception and eventually reduce repeat abortion.

Community based awareness programs to scale back unwanted pregnancies and encourage women to seek for help early was ranked highly with 28 points out of the possible 30 points. Unsafe abortion is a major consequence of unintended pregnancy mainly due to the high unmet need for contraception. Therefore, preventing unintended pregnancies by promoting contraceptive use would significantly reduce variety of unsafe abortions and maternal deaths [20]. Im- 
proving access to birth control information and services may be a main mechanism to scale back unintended pregnancies and consequently unsafe abortion. The activities associated with provision of contraception should be assessed to supply evidence on how they contribute to prevention of unsafe abortion [21]. Through counselling and provision of evidence-based, up-to-date information, providers can allow women to settle on contraception that they're going to feel most comfortable with, given their individual preferences and lifestyles.

In recent years, reductions in unplanned pregnancy have led to parallel reductions in unplanned childbearing, with positive benefits for women's health and children's well-being. But unplanned pregnancy rates remain high among unmarried women under the age of 30 , with majority of the pregnancies unintended [22]. Expanding coverage of post abortion care in the health facilities provides a transparent strategy for removing barriers to accessing post abortion contraception and suggests a path toward reducing rates of unintended pregnancies and unsafe abortions [23]. Evidence shows that legalizing abortion laws to permit services to be provided by skilled practitioners can reduce instances of abortion-related morbidity and mortality. The roles of research are vital in highlighting the importance of legalizing abortion laws.

\section{Conclusion}

Ten key research priorities in preventing unsafe abortion identified have the potential of providing direction on areas to focus on in preventing unsafe abortion. Until responsive interventions are taken, complications from unsafe abortion will remain a major cause of maternal mortality and morbidity. The research priority questions if implemented will help inform public dialogue to strengthen advocacy for safe abortion in the WHO Africa region.

\section{Authors Contribution}

NT, OL, BF, MS, DK, DS, AC and EH conceptualized the idea of the research prioritization exercise. KC, AM, TL, consolidated the online survey and provided technical support. All authors read and approved the final manuscript.

\section{Acknowledgements}

We thank all the experts who were involved in the research prioritization exercise for WHO Africa Region. They include: Zan Souleymane, Moufalilou Aboubakar, Bokossa Alexis Richard, Gaston D. Ahounou, Dina Gbenou, Hien Clotaire, Kiemtoré Sibraogo, Traoré Isidore Tiandiogo, Bassounda, Poïdigem, Dadjoari Moussa, Nguetabe Odile, Awad Adam Awad, Foumsou, Kini Brigitte Nsiku, Haimanot Ambelu Workineh, Tenaye Kebede, Girma Gemechu, Mekdes Daba, Deo Roseline, Samuel Oppong, Ivy Osei, Patrick K. Aboagye, Sy Telly, Férida Mara, Alpha Ahmadou Diallo, Tessougue Fatoumata, Idrissa Cissé, Sidy Diallo, Ben Moulaye Haidara, Moussa Kamissoko, Ojo Olumuyiwa, Ufere Joy, Ikpeze Okechukwu Christian, Chris Ega, Kabuteni Theopista John, Utumatwi- 
shima Jean Nepo, Ndaruhutse Victor, Ruzindana Kenneth, Mihretu Belete, Sithembile Dlamini-Nqeketo, K.S. Dithole, Rapinyana O., Ntombi Mtshali, Mercy Pindani, Azwihangwisi Helen Mavhandu-Mudzusi, Uta Lehmann, Boniface Ushie, David Norris, Blami Dao, Issiaka Sombie, Seni Kouanda, Richard Adanu,Goma Mboungou Mierette, Mavounia Ndoko Genil, Ngwenya, Mmamoitlamo and Mncwabe Pearl.

\section{Funding}

The WHO Regional office for Africa.

\section{Conflicts of Interest}

All authors declare no competing interests.

\section{References}

[1] Bearak, J., Popinchalk, A., Ganatra, B., Moller, A.-B., Tunçalp, Ö., Beavin, C., Kwok, L. and Alkema, L. (2020) Unintended Pregnancy and Abortion by Income, Region, and the Legal Status of Abortion: Estimates from a Comprehensive Model for 1990-2019. The Lancet Global Health, 8, e1152-e1161. https://doi.org/10.1016/S2214-109X(20)30315-6

[2] World Health Organization (2004) Unsafe Abortion: Global and Regional Estimates of the Incidence of Unsafe Abortion and Associated Mortality in 2000. WHO, Geneva.

[3] Say, L., Chou, D., Gemmill, A., et al. (2014) Global Causes of Maternal Death: A Who Systematic Analysis. The Lancet Global Health, 2, e323-e333.

[4] World Health Organization (2009) Women and Health: Today's Evidence, Tomorrow's Agenda. Geneva.

[5] Shah, I.H. and Åhman, E. (2012) Unsafe Abortion Differentials in 2008 by Age and Developing Country Region: High Burden among Young Women. Reproductive Health Matters, 20, 169-173. https://doi.org/10.1016/S0968-8080(12)39598-0

[6] World Health Organization (2015) Health Worker Roles in Providing Safe Abortion Care and Post-Abortion Contraception. World Health Organization, Geneva.

[7] Ganatra, B., Gerdts, C., Rossier, C., Johnson Jr., B.R., Tuncalp, Ö., Assifi, A., Sedgh, G., Singh, S., Bankole, A., Popinchalk, A., Bearak, J., Kang, Z. and Alkema, L. (2017) Global, Regional, and Subregional Classification of Abortions by Safety, 2010-14: Estimates from a Bayesian Hierarchical Model. The Lancet, 390, 2372-2381. https://doi.org/10.1016/S0140-6736(17)31794-4

[8] Myers, J.E. and Seif, M.W. (2010) Global Perspective of Legal Abortion-Trends, Analysis and Accessibility. Best Practice and Research Clinical Obstetrics and Gynaecology, 24, 457-466. https://doi.org/10.1016/j.bpobgyn.2010.04.002

[9] World Health Organization (2012) Safe Abortion: Technical and Policy Guidance for Health Systems. World Health Organization, Geneva.

[10] Bongaarts, J. (2011) Can Family Planning Programs Reduce High Desired Family Size in Sub-Saharan Africa? International Perspectives on Sexual and Reproductive Health, 37, 209-216. https://doi.org/10.1363/3720911

[11] Sedgh, G., Singh, S. and Hussain, R. (2014) Intended and Unintended Pregnancies Worldwide in 2012 and Recent Trends. Studies in Family Planning, 45, 301-314. 
https://doi.org/10.1111/j.1728-4465.2014.00393.x

[12] United Nations (2015) Transforming Our World: The 2030 Agenda for Sustainable Development. United Nations, New York.

[13] Barnard, S., Kim, C., Park, M.H., et al. (2015) Doctors or Mid-Level Providers for Abortion. Cochrane Database of Systematic Reviews, No. 7, CD011242. https://doi.org/10.1002/14651858.CD011242

[14] United Nations (2015) Every Woman Every Child. Saving Lives, Protecting Futures: Progress Report on the Global Strategy for Women's and Children's Health. New York.

[15] Fleurence, R.L. and Torgerson, D.J. (2004) Setting Priorities for Research. Health Policy, 69, 1-10. https://doi.org/10.1016/j.healthpol.2003.11.002

[16] Rudan, I., Chopra, M., Kapiriri, L., Gibson, J., Lansang, M.A., et al. (2008) Setting Priorities in Global Child Health Research Investments: Universal Challenges and Conceptual Framework. Croatian Medical Journal, 49, 398-408. https://doi.org/10.3325/cmj.2008.3.307

[17] Ali, M., Farron, M., Quedraogo, L., Mahaini, R.K., Miller, K. and Kabra, R. (2018) Research Gaps and Emerging Priorities in Sexual and Reproductive Health in Africa and Eastern Mediterranean Regions. Reproductive Health Journal, 15, 39. https://doi.org/10.1186/s12978-018-0484-9

[18] Mendis, S. and Alwan, A. (2011) Prioritized Research Agenda for Prevention and Control of Noncommunicable Diseases. World Health Organization, Geneva.

[19] Souza, J.P., Widmer, M., Gülmezoglu, A.M., Lawrie, T.A., Adejuyigbe, E.A., Carroli, G., Crowther, C., Currie, S.M., Dowswell, T., Hofmeyr, J., et al. (2014) Maternal and Perinatal Health Research Priorities beyond 2015: An International Survey and Prioritization Exercise. Reproductive Health, 11, Article No. 61.

https://doi.org/10.1186/1742-4755-11-61

[20] Grimes, D.A., Benson, J., Singh, S., Romero, M., Ganatra, B., Okonofua, F.E. and Shah, I.H. (2006) Unsafe Abortion: The Preventable Pandemic. The Lancet: Sexual and Reproductive Health, 368, 1908-1919. https://doi.org/10.1016/S0140-6736(06)69481-6

[21] Ministry of Health, Kenya (2013) Key Findings of a National Study: Incidence and Complications of Unsafe Abortion in Kenya. Women's Lives Matter Preventing Unsafe Abortion in Kenya.

[22] Faundes, A., Comendant, R., Dilbaz, B., Jaldesa, G., Leke, R., Mukherjee, B., de Gil, M.P. and Tavara, L. (2020) Preventing Unsafe Abortion: Achievements and Challenges of a Global FIGO Initiative. Best Practice and Research Clinical Obstetrics and Gynaecology, 62, 101-112. https://doi.org/10.1016/j.bpobgyn.2019.05.016

[23] Karpilow, Q., Manlove, J., Sawhill, I. and Thomas, A. (2013) The Role of Contraception in Preventing Abortion, Nonmarital Childbearing, and Child Poverty. Brookings (Blog).

https://www.brookings.edu/research/the-role-of-contraception-in-preventing-abort ion-nonmarital-childbearing-and-child-poverty 


\section{Abbreviations}

CHNRI: Child health and nutrition research initiatives; WHO: World Health Organization; UHC: Universal health coverage; SRHR: sexual and reproductive health and rights, EMRO: World Health Organization Office for the Eastern Mediterranean; AFRO: World Health Organization Regional Office for Africa. 